\title{
On the Performance Limitations of Realistic Massive MIMO Deployments in 5G mmWave Wireless Cellular Networks
}

\author{
Spyros Lavdas ${ }^{1}$, Panagiotis Gkonis ${ }^{2}$, Panagiotis Trakadas ${ }^{2}$ and Lambros Sarakis ${ }^{2}$ \\ ${ }^{1}$ Department of Computer Science, Neapolis University, Paphos, 8042,Cyprus. E-mail: s.lavdas@nup.ac.cy \\ ${ }^{2}$ General Department, National and Kapodistrian University of Athens, Sterea Ellada, 34400 Dirfies Messapies, Greece. \\ E-mails: \{pgkonis,ptrakadas,lsarakis\}@uoa.gr
}

\begin{abstract}
In this paper the performance of realistic massive multiple input multiple output configurations operating at the millimeter wave frequency band is evaluated. Performance is evaluated statistically by executing a sufficient number of Monte Carlo simulations with the help of a developed semi-static simulator in a fifth-generation multicellular orientation. According to the results, although the increase in the number of radiating elements potentially leads to improved performance metrics (e.g., increased throughput, reduced blocking probability and transmission power), the deployment of an increased number of highly directional beams in predefined angular locations can lead to performance degradation. In particular, for 128 radiating elements and 8 directional beams per base station (BS), total throughput can reach 2320 Mbps with an equivalent transmission power per BS less than $2 \mathrm{~W}$. However, system performance in terms of accepted users and blocking probability can deteriorate significantly when considering an equivalent grid of beams with 16 active sectors.
\end{abstract}

Keywords-5G, massive MIMO, mmWave transmission, System level simulations

\section{INTRODUCTION}

The full deployment of fifth-generation (5G) networks has a three-fold goal of service support: enhanced mobile broadband (eMBB), ultra reliable low latency communications (URLLC) as well as massive machine type communications (mMTC) [1]-[2]. In this context, novel technologies have been introduced in order to support increased data rates and low latency applications along with more efficient spectrum utilization via the new concept of heterogeneous networks [3]: millimeter wave (mmWave) transmission [4], non-orthogonal multiple access (NOMA) [5] as well as massive multiple input multiple output (MIMO) systems [6]-[7]. In the latter case, an increased number of transmit antennas (typically much larger compared to the number of active users within a geographical area) is deployed. Hence, a sufficient number of directional beams can be generated towards the positions of active users.

To this end, the proper deployment and performance limitations of massive MIMO systems have attracted scientific interest over the last years. In [8], the authors show that the spectral efficiency of such systems does increase with the increase in the number of antennas installed at the Base Station (BS). In [9], one of the key findings is that Cell Free (CF) massive MIMO systems provide better coverage than conventional collocated ones and uncoordinated small cells. The work in [10] is focused on efficient signal detection techniques for massive MIMO configurations. In [11], downlink multi-user mmWave massive MIMO systems in both centralized and distributed schemes have been considered. Performance was evaluated in terms of spectral efficiencies, while the impact of the number of total transmit antennas, the number of users and the location of the distributed antenna arrays on system performance was evaluated as well. In [12], the performance of a Time-division duplex (TDD)-based massive MIMO deployment scenario has been evaluated. According to the presented results, TDDbased massive MIMO in $10 \mathrm{MHz}$ bandwidth reveals up to $212 \%$ and $50 \%$ higher cell throughput compared to Frequency-division duplex (FDD)-based MIMO deployments with $10 \mathrm{MHz}$ and $20 \mathrm{MHz}$ bandwidth, respectively. In [13], a survey on current commercial massive MIMO products is provided, as well as system-level simulation results.

In all the aforementioned studies however, either limited network topologies have been considered (i.e., single cell scenarios), or limited number of active users. In this paper, the goal is to evaluate the performance of realistic antenna configurations in $5 \mathrm{G}$ wireless networks. The novelty of our work can be summarized as follows: a) Development of massive MIMO antenna radiation patterns operating at 28 $\mathrm{GHz}$ b) Utilization of a circular array comprised of vertical arrays that use separate feeding ports leading to a reduced complexity of RF-chain precoder c) Performance evaluation of the aforementioned geometry in multicellular/multiuser $5 \mathrm{G}$ networks d) Channel modeling according to 3GPP specifications. The rest of this paper is organized as follows. In Section II, the MIMO channel model for $5 \mathrm{G}$ configurations is described. Simulation framework is analyzed in Section III, while antenna configurations are described in Section IV. Simulations results are presented and discussed in Section V, while concluding remarks are outlined in Section VI.

\section{MIMO CHANNEL MODELLING IN 5G WIRELESS CELLULAR NETWORKS}

The latest 3GPP channel model (3GPP TR38.901) [14] supports a wide frequency range $(0.5-100 \mathrm{GHz})$ and a large bandwidth (up to $10 \%$ of carrier frequency). To this end, the channel coefficient for an arbitrary pair of transmit-receive antennas $(t x-r x)$ can be expressed as a sum of individual components from $N$ clusters and $M$ subpaths per cluster. For a specific cluster, the channel is formulated as follows:

$$
\begin{aligned}
H_{u, s, n}^{\mathrm{NLOS}}=\sqrt{\frac{P}{M}} \sum_{m=1}^{M} \mathbf{F}_{r x, n, m}^{\mathrm{T}} \boldsymbol{\Theta}_{n, m} \mathbf{F}_{t x, n, m} \exp \left(\frac{j 2 \pi\left(\hat{r}_{r x, n, m}^{\mathrm{T}} \cdot \bar{d}_{r x, u}\right)}{\lambda}\right) \exp \left(\frac{j 2 \pi\left(\hat{r}_{t x, n, m}^{\mathrm{T}} \cdot \bar{d}_{t x, s}\right)}{\lambda}\right) \\
\mathbf{F}_{r x, n, m}=\left[\begin{array}{l}
F_{r x, u, \theta}\left(\theta_{n, m, Z O A}, \phi_{n, m, A O A}\right) \\
F_{r x, u, \phi}\left(\theta_{n, m, Z O A}, \phi_{n, m, A O A}\right)
\end{array}\right] \\
\mathbf{F}_{t x, n, m}=\left[\begin{array}{l}
F_{t x, \mathrm{~s}, \theta}\left(\theta_{n, m, Z O D}, \phi_{n, m, A O D}\right) \\
F_{t x, \mathrm{~s}, \phi}\left(\theta_{n, m, Z O D}, \phi_{n, m, A O D}\right)
\end{array}\right]
\end{aligned}
$$




$$
\boldsymbol{\Theta}_{n, m}=\left[\begin{array}{lr}
\exp \left(j \Phi_{n, m}^{\theta \theta}\right) & \sqrt{\kappa_{n, m}{ }^{-1}} \exp \left(j \Phi_{n, m}^{\theta \phi}\right) \\
\sqrt{\kappa_{n, m}{ }^{-1}} \exp \left(j \Phi_{n, m}^{\phi \theta}\right) & \exp \left(j \Phi_{n, m}^{\phi}\right)
\end{array}\right]
$$

In (2)-(3), $\theta_{n, m, Z o D}$ and $\theta_{n, m, Z o A}$ represent the angles of departure (AoD) and arrival (AoA), respectively, in the vertical plane for the $m^{\text {th }}$ subpath $(1 \leq m \leq M)$ of the $n^{\text {th }}$ cluster $(1 \leq n \leq N)$. The corresponding parameters for the horizontal plane are $\phi_{n, m, A o D}$ and $\phi_{n, m, A o A}$, respectively. Moreover, $P_{n}$ is the power of the $n^{\text {th }}$ cluster, set $\left\{\Phi_{n, m}^{\theta \theta}, \Phi_{n, m}^{\theta \phi}, \Phi_{n, m}^{\phi \theta}, \Phi_{n, m}^{\phi \phi}\right\}$ in (4) corresponds to initial phases uniformly distributed in $(-\pi, \pi)$ while $\kappa_{n, m}$ parameter is the generated cross polarization power ratio (XPR) for each ray $m$ of cluster $n$. In addition, $\lambda$ is the carrier wavelength, $\hat{r}_{r x, n, m}$ is the spherical unit vector with azimuth arrival angle $\phi_{n, m, A O A}$ and elevation arrival angle $\theta_{n, m, Z O A}$, while $\hat{r}_{t x, n, m}$ is the spherical unit vector with azimuth departure angle $\phi_{n, m, A O D}$ and elevation departure angle $\theta_{n, m, Z O D}$. Moreover, $\mathbf{F}_{t x, n, m} / \mathbf{F}_{r x, n, m}$ represent the field pattern of transmit/receive antenna element $s / u$, respectively $\left(1 \leq s \leq N_{t}, 1 \leq u \leq N_{r}\right), \bar{d}_{r x, u}$ is the location vector of receive antenna element $u$ and $\bar{d}_{t x, \mathrm{~s}}$ is the location vector of transmit antenna element $s$. Finally, $\mathbf{A}^{\mathrm{T}}$ denotes the transpose of matrix $\mathbf{A}$ and $j$ the imaginary unit.

The overall geometry is depicted in Fig. 1, considering only the x-y plane. The BS and Mobile Station (MS) array orientation is represented by angles $\Omega_{B S} / \Omega_{M S}$, respectively. In addition, $\theta_{B S}$ is the Line of Sight (LOS) AoD direction between the BS and MS (with respect to the broadside of the BS array), while $\theta_{M S}$ is the angle between the BS-MS LOS and the MS broadside. Finally, $\Delta_{n, m, A o D}$ is the angle offset of the $m^{\text {th }}$ subpath with respect to $\theta_{n, m, A o D}$ and $\triangle_{n, m, A o A}$ the corresponding offset with respect to $\theta_{n, m, A o A}$.

\section{SIMULATION FRAMEWORK}

A system-level simulator has been developed, executing a sufficient number of Monte Carlo (MC) simulations in multicellular wireless orientations. MSs enter the network sequentially, following a uniform distribution. It is assumed that during each MC run, the positions of the MSs remain unchanged (semi-static simulator). For each candidate MS, all associated system level parameters (e.g. pathlosses, shadowing, MIMO channel matrices) are calculated and power allocation per requested subcarrier takes place. In this context, considering an arbitrary MIMO configuration with $N_{t}$ transmit antennas and $N_{r}$ receive antennas $\left(N_{t} \times N_{r}\right)$, the equivalent channel matrix per subcarrier is calculated after the summation of all individual cluster components of (1) for all $t x-r x$ pairs (subcarriers are grouped in Physical Resource Blocks-RPBs [15], defined as 12 consecutive subcarriers in the frequency domain). If the requested power per subcarrier exceeds a predefined threshold, then the candidate MS is rejected from the network. The MC run comes to an end when there is an excess of BS power or lack of available subcarriers.

A flowchart of the overall procedure is shown in Fig. 2, while all simulation parameters are summarized in Table I (DTP is the downlink transmission power of the new MS). In all simulation scenarios, as it will be described in Section $\mathrm{V}$, three Key Performance Indicators (KPIs) have been considered: Total network throughput, blocking probability $(B P$, defined as the number of MSs that successfully enter the network to the total number of MSs requesting access) as well as total downlink transmission power from all BSs. Simulation setup and parameter selection are aligned with most of related works described in [16], regarding system- and link- level simulations.

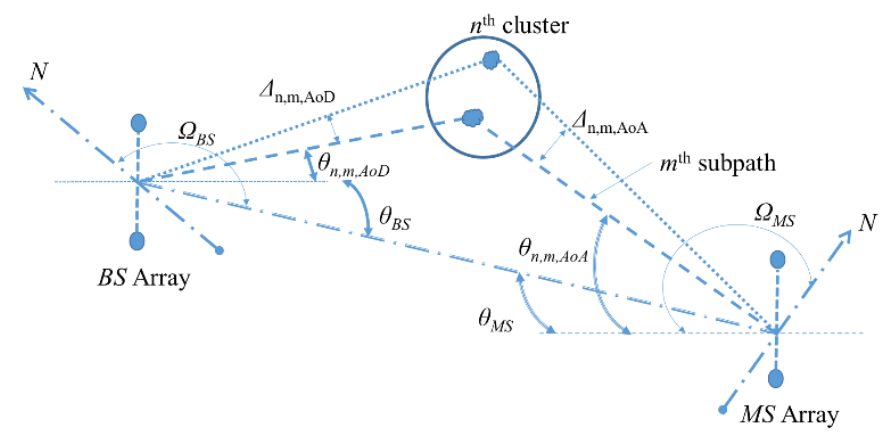

Fig. 1. 3GPP channel model for $5 \mathrm{G}$ wireless systems

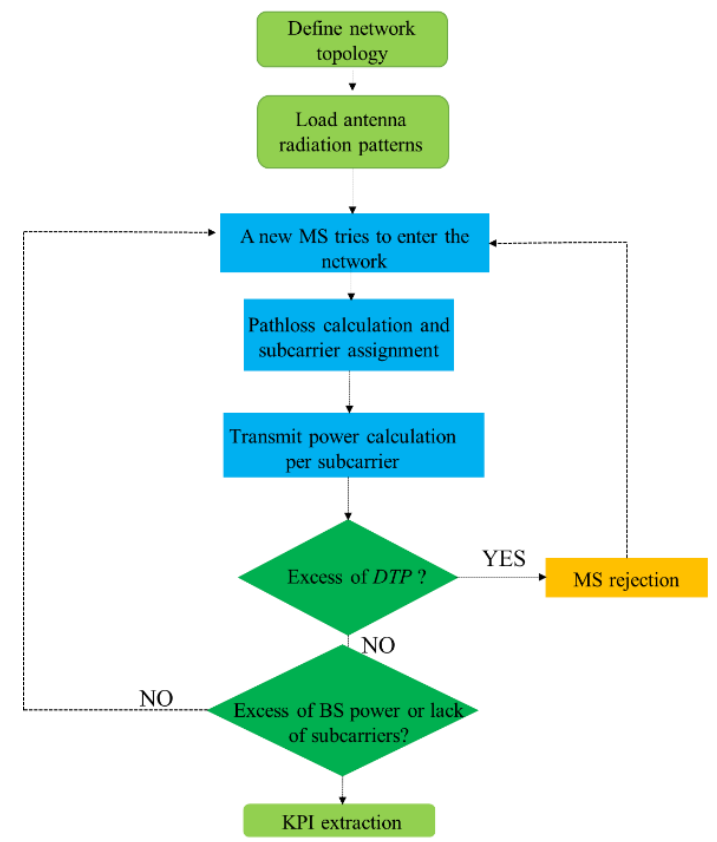

Fig. 2. Flowchart of the 5G MIMO System-Level Simulator TABLE I. SIMULATION PARAMETERS

\begin{tabular}{|c|c|}
\hline Parameter & Value/Assumption \\
\hline Tiers of cells around the central cell/Number of cells & $2 / 19$ \\
\hline Pathloss model & $\begin{array}{l}\text { Urban Macro- } \\
\text { cellular (UMa) }\end{array}$ \\
\hline Carrier frequency $(\mathrm{GHz})$ & 28 \\
\hline Channel Bandwidth (MHz) & 100 \\
\hline Subcarrier spacing $(\mathrm{kHz})$ & 60 \\
\hline PRBs per BS & 132 \\
\hline Subcarriers per PRB & 12 \\
\hline Cell radius $(m)$ & 500 \\
\hline Assigned PRBs per MS and equivalent data rates & $\begin{array}{c}5 \text { (7.2Mbps) and } \\
15(21.6 \mathrm{Mbps})\end{array}$ \\
\hline Monte Carlo snapshots per scenario & $10^{4}$ \\
\hline Required $E_{b} / N_{o}(\mathrm{~dB})$ & 9.6 \\
\hline Maximum transmission power per BS/MS (W) & $20 / 1$ \\
\hline Antenna elements per MS & 2 \\
\hline
\end{tabular}




\section{ANTENNA CONFIGURATION}

An attractive massive MIMO antenna configuration is characterized by the use of a multitude of dual polarized (DP) or circular polarized (CP) antennas which are horizontally or vertically separated. Such type of antenna schemes can utilize both the spatial and polarization dimensions [17]-[21]. Besides, the ever-growing interest in the exploitation of mmWave domain dictates the installation of millimeter $\mathrm{DP} / \mathrm{CP}$ antennas in a MIMO system.

The former requirements for a MIMO scheme can be easily achieved with the most common type of CP antenna, namely the crossed dipole antenna, with an RF- to millimeter wave frequency range [22]-[25]. In addition, such type of antennas can enhance in a more efficient way the diversity gain of the receiver, especially in cases of arriving signals with different power compared to the respective limited diversity gain coming from DP antennas [26]-[27]. By an appropriate exploitation of the left- and right-hand polarization, the dipole crossed array can receive all orientations of signals eliminating the multipath fading of a rich propagation environment. Thus, MIMO transmission and reception holds an immutable behavior in terms of the $\mathrm{CP}$ antenna positioning [28].

In the present study, a reflector using as an exciter a crossed dipole antenna has been designed to resonate at the mmWave carrier frequency of $28 \mathrm{GHz}$ as shown in Fig. 3. In particular, the crossed dipole consists of two identical but orthogonal $\left( \pm 45^{\circ}\right)$ half-wave dipoles. The crossed dipoles are fed by two separates ports with the requisite $90^{\circ}$ phase difference between them. Finally, the width and the spacing between the two radiating elements are of the order of $\lambda / 100$ [29], as shown on the right part (profile of the crossed dipole) of Fig. 3. Note here that utilization of separate feeding ports facilitates the development of beamforming-oriented topologies when it is necessary.

The current work utilizes different types of crossed dipole arrays in order to study the efficiency of different MIMO configurations. To be more specific, each antenna array consists of $w$ crossed dipoles equally spaced on a ring $\left(\alpha=360^{\circ} / w\right)$ with diameter $q$ measured in units of $\lambda$ as presented in Fig. 4. Besides, $v$ crossed dipoles are vertically located building a block of antennas [30]-[31]. In order to enhance the gain in the horizontal plane each of the vertical crossed dipoles is placed $\lambda / 2$ far apart. To generate unidirectional $\mathrm{CP}$ radiation, all the radiating elements are also placed above a perfect electric conductor (PEC) reflector in a distance of $\sim \lambda / 4$ leading to a further gain increase [29].

It should be mentioned that all developed circular array configurations are characterized by the $w, v, q$ and $a$ parameters unless otherwise stated, Fig. 4. In addition, all the field properties of the circular arrays such as far-field radiation pattern have been computed by the method of moments (MoM) [32]. Note here that the 3-D computational model, namely full wave model, developed for our simulation results has taken into account the excitation of all mutual currents among all the radiating elements of the circular arrays. Thus, all the changes in the radiation pattern and input impedance of the developed antenna configurations due to the mutual coupling among the radiating elements have been taken into consideration [33].

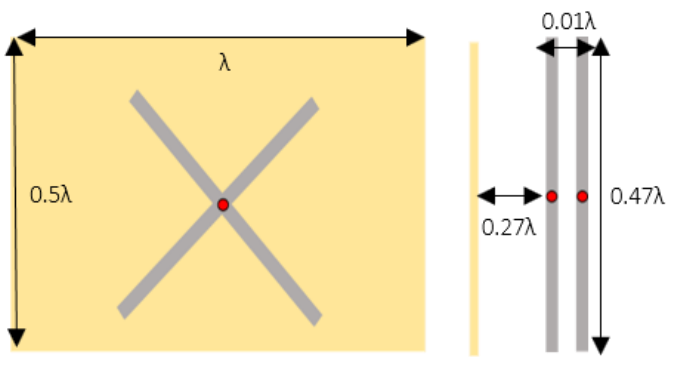

Fig. 3. Geometry of the reflector using a crossed dipole as an exciter

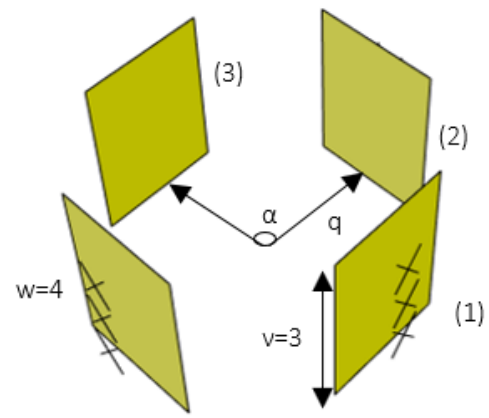

Fig. 4. An example of circular array. This geometry consists of $12(v \times w)$ crossed half-wave dipoles ( 24 radiating elements) uniformly distributed, $a=360 / 4=90^{\circ}$, with a ring radius $q$

Finally, the antenna configurations presented in Fig. 5 consist of lossless crossed dipoles without any matching network between the antenna and the source as such type of analysis is beyond the scope of the current paper. Thus, the directivity of the circular array corresponds to the total gain as there are not any mismatches due to reflections between the crossed dipoles and the corresponding feeding points [29].

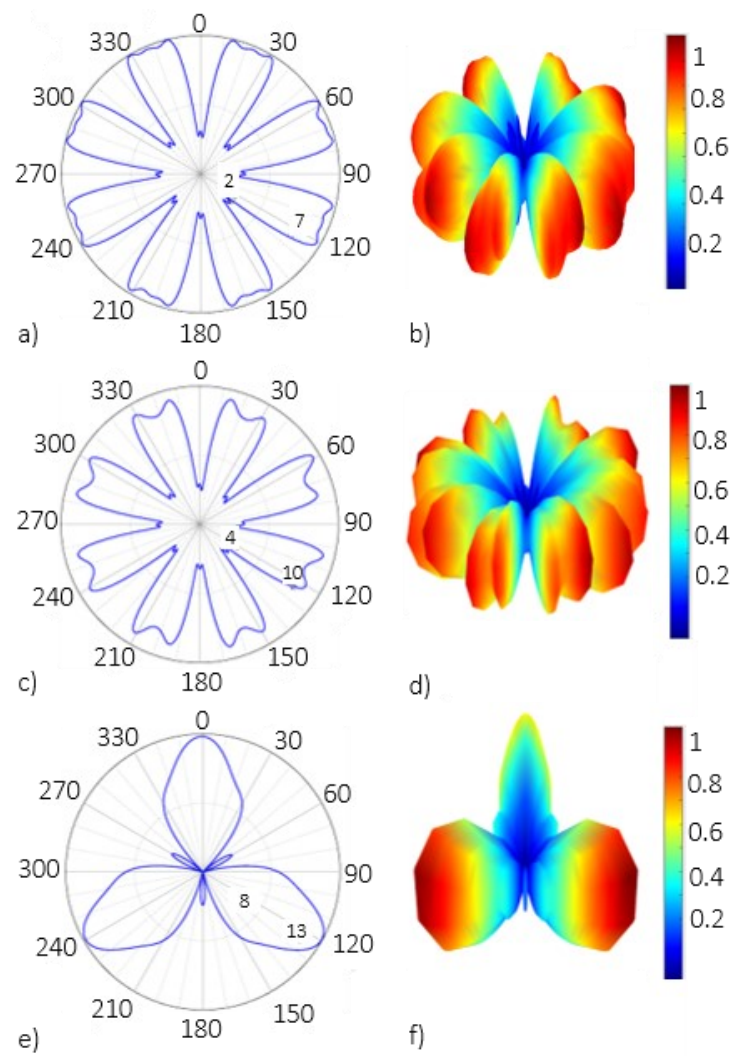

Fig. 5. Gain on the horizontal plane (azimuth) and 3D normalized power pattern for three different crosssed dipole configurations 
Fig. 5 shows the gain on the azimuth plane as well the 3D gain pattern of three different array geometries. In particular, the results of Fig. $5(\mathrm{a}, \mathrm{b})$ correspond to the parameters $w=8$, $v=4, \alpha=45^{\circ}, q=2.1 \lambda$ leading to a maximum directivity of 6.97 $\mathrm{dB}$ with Half Power Beam Width $(H P B W)$ equal to $31^{\circ}$. By keeping the parameters $w, a, q$ constant and increasing $v$ to 8 , the maximum achieved gain on the horizontal plane is raised to $10.4 \mathrm{~dB}$ as expected, Fig. $5(\mathrm{c}, \mathrm{d})$. Interestingly enough the $H P B W$ attains the value of $31^{\circ}$. Following the concept of low power tiny $\mathrm{BSs}$, a 3 -sector mmWave $\mathrm{BS}$ is designed and simulated with the following parameters: $w=3, v=10, \alpha=120^{\circ}$, $q=0.81 \lambda$. The beamforming behavior of the array is also obvious, while the maximum gain obtained is $13.4 \mathrm{~dB}$ as shown in Fig. 5 (e, f). The results of Fig. 5 are necessary for the calculation of Cumulative Distribution Functions (CDF curves) presented in the next section.

\section{RESULTS}

Simulation results (CDF curves) are presented in Figs. 6 8 , for the considered KPIs of section III and four massive MIMO configurations: $10 \times 3 \quad(3$ sectors, 60 radiating elements), $4 \times 8$ ( 8 sectors, 64 radiating elements), $8 \times 8$ ( 8 sectors, 128 radiating elements) and $8 \times 16$ (16 sectors, 256 radiating elements), where the number of sectors corresponds to the total number of generated beams from the antenna configuration at BSs, as shown in Fig. 5. The third index in each figure legend corresponds to the allocated PRBs per MS. In all cases, diversity combining transmission mode has been assumed. Moreover, in all the examined scenarios two tiers of cells around the central cell have been considered.

As it can be observed from Fig. 6, in the first three configurations throughput is maximized for 5 allocated PRBs per MS. In this case, corresponding mean values are $2324 / 2320 / 2238 \mathrm{Mbps}$ for the $4 \times 8 / 8 \times 8 / 10 \times 3$ configurations, respectively, as shown in Fig. 5. In all the examined scenarios throughput is reduced for 15 PRBs per MS, as expected, since in this case a reduced number of high data rate MSs can be supported. In Fig. 7, the total downlink transmission power for the aforementioned configurations is depicted. As it can be observed, in the $8 \times 8 \mathrm{MIMO}$ case (Fig. 5 (c,d)) a significant downlink transmission power reduction can be achieved compared to the $4 \times 8$ case (Fig. 5 (a,b)). In particular, mean transmission power is $65 / 34 \mathrm{~W}$ in the $4 \times 8 / 8 \times 8 \mathrm{MIMO}$ configuration, respectively. This power is further reduced to $19 \mathrm{~W}$ (i.e., $1 \mathrm{~W}$ per BS) in the $10 \times 3$ configuration (Fig. $5(\mathrm{e}, \mathrm{f})$ ). In this context, it is interesting to note that apart from downlink transmission power, $B P$ is minimized as well: mean value is limited to $1 \%$, while in the $8 \times 8$ case corresponding value is $2 \%$.

However, as it becomes apparent from all Figs., in the $8 \times 16$ configuration all KPIs can significantly deteriorate compared to the previous cases. Therefore, the deployment of many directional beams with an equivalent increase in the number of transmit antennas does not always result in reduced transmission power and $B P$. In the $10 \times 3$ case, spatial coverage is improved, leading to an equivalent improvement of the examined KPIs, despite the fact that in this case we have the fewest number of radiating elements compared to the other configurations.

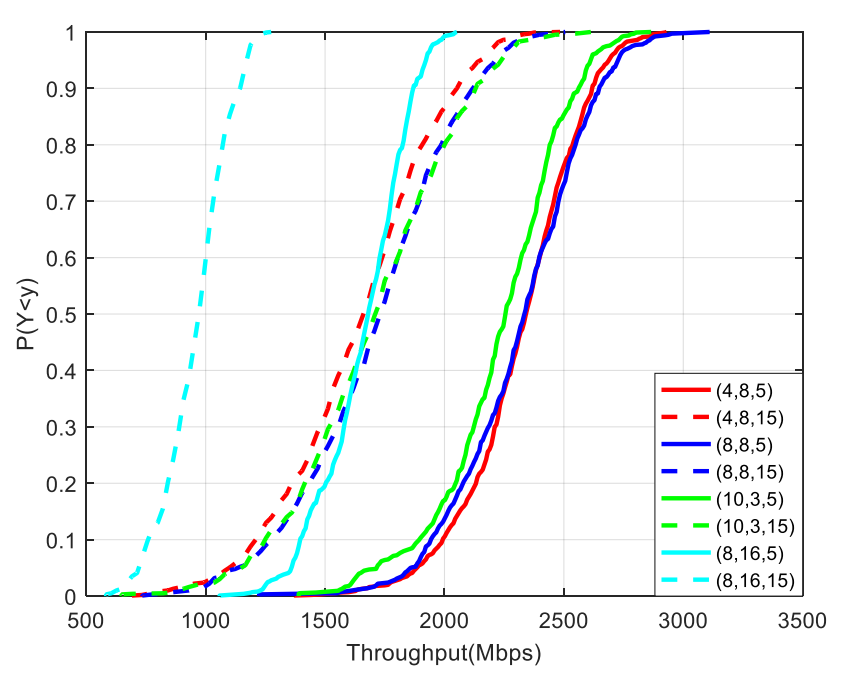

Fig. 6. Total network throughput for various MIMO configurations (CDF curves)

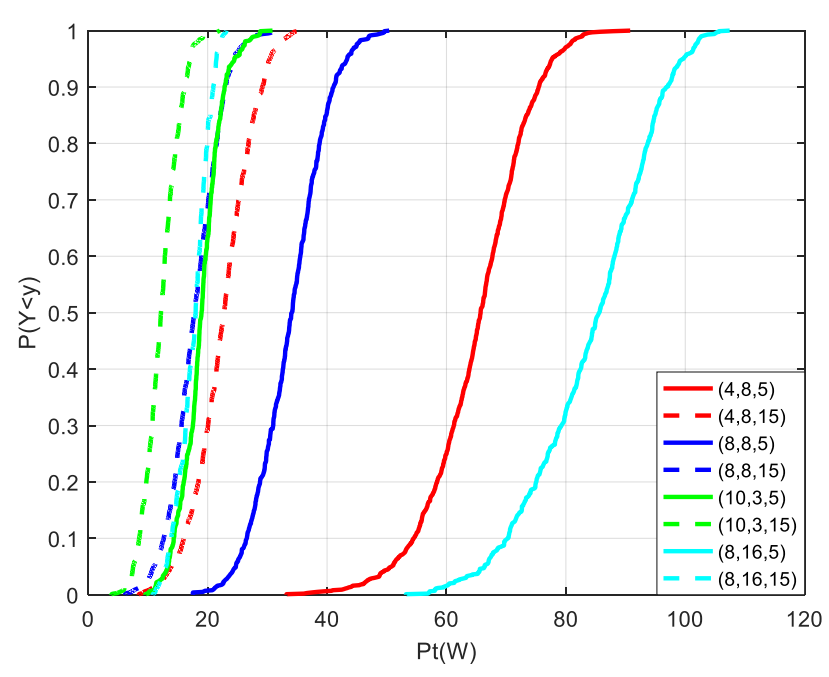

Fig. 7. Total transmission power for various MIMO configurations (CDF curves)

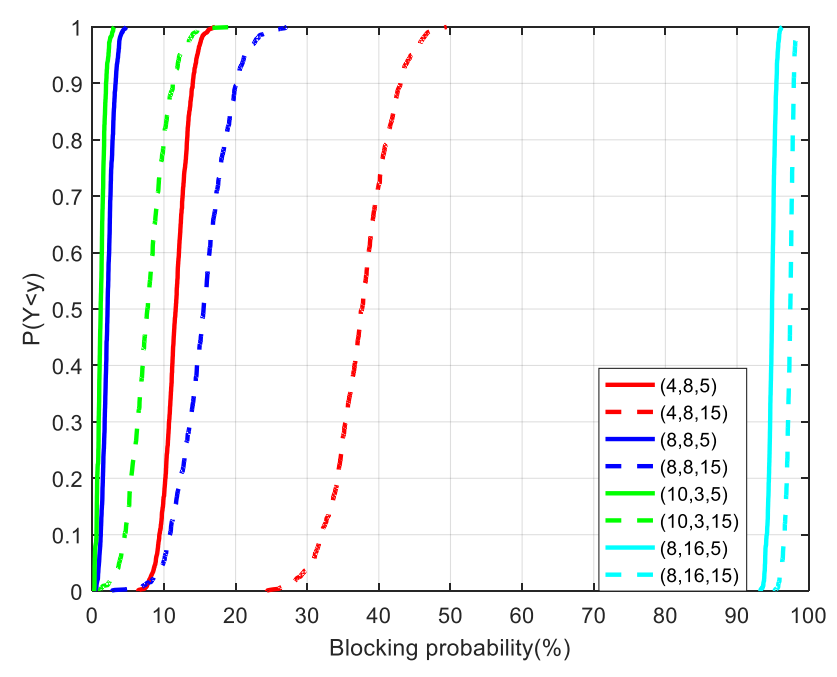

Fig. 8. Blocking probability for various MIMO configurations (CDF curves) 


\section{CONCLUSIONS}

The performance of various realistic massive MIMO configurations has been evaluated, in the context of $5 \mathrm{G}$ mmWave wireless cellular networks. Extensive system-level simulations were performed, in network topologies with two tiers of cells around the central cell. According to the presented results, the deployment of an increased number of highly directional beams in predefined angular locations does not result in improved performance metrics. On the contrary, the deployment of sectors with increased beamwidth leads to downlink transmission power and blocking probability reduction. These results are particularly important towards the design and implementation of realistic 5G deployments, since improved KPIs can be achieved with minimum signaling burden and transceiver configurations (reduced number of dedicated pilot signals per BS for optimum beam selection).

\section{ACKNOWLEDGMENT}

This work has been partially supported by the Affordable $5 \mathrm{G}$ project, funded by the European Commission under Grant Agreement H2020-ICT-2020-1, number 957317 through the Horizon 2020 and 5G-PPP programs (www.affordable5g.eu/).

\section{REFERENCES}

1] P. Popovski, K. F. Trillingsgaard, O. Simeone and G. Durisi, " $5 \mathrm{G}$ Wireless Network Slicing for eMBB, URLLC, and mMTC: A Communication-Theoretic View," IEEE Access, vol. 6, pp. 5576555779, 2018, doi: 10.1109/ACCESS.2018.2872781.

[2] A. K. Bairagi et al., "Coexistence Mechanism between eMBB and uRLLC in 5G Wireless Networks," IEEE Transactions on Communications, vol. 69, no. 3, pp. 1736-1749, March 2021, doi: 10.1109/TCOMM.2020.3040307.

[3] Y. Xu, G. Gui, H. Gacanin and F. Adachi, "A Survey on Resource Allocation for 5G Heterogeneous Networks: Current Research, Future Trends, and Challenges," IEEE Communications Surveys \& Tutorials, vol. 23, no. 2, pp. 668-695, Secondquarter 2021, doi: 10.1109/COMST.2021.3059896.

4] A. N. Uwaechia and N. M. Mahyuddin, "A Comprehensive Survey on Millimeter Wave Communications for Fifth-Generation Wireless Networks: Feasibility and Challenges," IEEE Access, vol. 8, pp. 6236762414, 2020, doi: 10.1109/ACCESS.2020.2984204.

[5] N. Nomikos, E. T. Michailidis, P. Trakadas, D. Vouyioukas, T. Zahariadis and I. Krikidis, "Flex-NOMA: Exploiting Buffer-Aided Relay Selection for Massive Connectivity in the 5G Uplink," IEEE Access, vol. 7, pp. 88743-88755, 2019, doi: 10.1109/ACCESS.2019.2926770.

[6] H. Ji et al., "Overview of Full-Dimension MIMO in LTE-Advanced Pro," IEEE Communications Magazine, vol. 55, no. 2, pp. 176-184, February 2017, doi: 10.1109/MCOM.2016.1500743RP.

[7] M. A. Albreem, M. Juntti and S. Shahabuddin, "Massive MIMO Detection Techniques: A Survey," IEEE Communications Surveys \& Tutorials, vol. 21, no. 4, pp. 3109-3132, Fourthquarter 2019, doi: 10.1109/COMST.2019.2935810.

[8] M. Gahadza and S. Winberg, "Performance of massive MIMO Systems for Future Generation Wireless Systems," IEEE $10^{\text {th }}$ International Conference on Mechanical and Intelligent Manufacturing Technologies (ICMIMT), Cape Town, South Africa, 2019, pp. 204-211, doi: 10.1109/ICMIMT.2019.8712054.

[9] J. Zhang, S. Chen, Y. Lin, J. Zheng, B. Ai and L. Hanzo, "Cell-Free Massive MIMO: A New Next-Generation Paradigm," IEEE Access, vol. 7, pp. 99878-99888, 2019, doi: 10.1109/ACCESS.2019.2930208.

[10] M. A. Albreem, M. Juntti and S. Shahabuddin, "Massive MIMO Detection Techniques: A Survey," IEEE Communications Surveys \& Tutorials, vol. 21, no. 4, pp. 3109-3132, Fourthquarter 2019, doi: 10.1109/COMST.2019.2935810.

11] J. Li, D. Yue and Y. Sun, "Performance Analysis of Millimeter Wave Massive MIMO Systems in Centralized and Distributed Schemes," IEEE Access, vol. 6, pp. 75482-75494, 2018, doi: 10.1109/ACCESS.2018.2882003.

[12] E. Zeydan, O. Dedeoglu and Y. Turk, "Experimental Evaluations of TDD-Based Massive MIMO Deployment for Mobile Network
Operators," IEEE Access, vol. 8, pp. 33202-33214, 2020, doi: 10.1109/ACCESS.2020.2974277.

[13] J. Guo, N. Li, Z. Jiang, S. Liu and P. Chen, "System-level Evaluation on Practical Massive MIMO Deployment Scenarios for 5G," IEEE $5^{\text {th }}$ International Conference on Computer and Communications (ICCC), Chengdu, China, 2019, pp. 993-998, doi: 10.1109/ICCC47050.2019.9064056.

[14] 3GPP TR 38.901 Version 14.3.0 Rel. 14, Study on Channel Model for Frequencies from 0.5 to $100 \mathrm{GHz}, 2018$.

[15] 3GPP TS 138211 Version 15.3.0 Rel.15, 5G NR Physical channels and modulation, 2018

[16] P. K. Gkonis, P. T. Trakadas and D. I. Kaklamani, "A Comprehensive Study on Simulation Techniques for 5G Networks: State of the Art, Analysis and Future Challenges," Electronics (MDPI), vol. 9, no. 3, 2020, doi: 10.3390/electronics9030468.

[17] E. Bjornson, L. Sanguinetti, H. Wymeersch, J. Hoydis and T. L. Marzetta, "Massive MIMO is a Reality- What is next? Five Promising Research Directions for Antenna Arrays," Digital Signal Processing, vol. 94, pp. 3-20, 2019, doi: 10.1016/j.dsp.2019.06.007.

[18] R. Chataut and R. Akl, "Massive MIMO Systems for 5G and beyond Networks- Overview, Recent Trends, Challenges, and Future Research Direction," Sensors $(M D P I)$, vol. 20, no. 10, 2020, doi: $10.3390 / \mathrm{s} 20102753$

[19] E. Bjornson, J. Hoydis and L. Sanguinetti, "Massive MIMO Networks: Spectral, Energy, and Hardware Efficiency," Pub. Now Foundations and Trends, 2017, doi: 10.1561/2000000093.

[20] A. Czylwik and A. Dekorsy, "System-Level Performance of Antenna Arrays in CDMA-Based Cellular Mobile Radio Systems," EURASIP Journal on Advances in Signal Processing, 2004, doi: $10.1155 / \mathrm{S} 111086570440403 \mathrm{X}$.

[21] R. B. Shaik and G. Sasikala, "Performance Improvement of the Base Station Antenna by Using MIMO in Mobile Communications," Journal of Critical Reviews, vol. 7, no. 4, pp. 243-251, 2020, doi: 10.31838/jcr.07.04.45

[22] S. X. Ta, I. Park and R. W. Ziolkowski, "Crossed Dipole Antennas: A review," IEEE Antennas and Propagation Magazine, vol. 57, no. 5, pp. 107-122, 2015, doi: 10.1109/MAP.2015.2470680.

[23] H. Tang, X. Zong and Z. Nie, "Novel Broadband Dual-Polarized Antenna for 5G Applications," International Symposium on Antennas and Propagation (ISAP), Busan, Korea (South), 2018, pp. 1-2.

[24] K. Honda, T. Fukushima and K. Ogawa, "Full-Azimuth Beam Steering MIMO Antenna Arranged in a Daisy Chain Array Structure," Micromachines, vol. 11, 2020, doi: 10.3390/mi11090871.

[25] V. R. Rentapalli and Z. J. Khan, "MIMO and smart antenna technologies for 3G and 4G," Information Techology and Mobile Communication, vol. 147 Springer, Berlin, doi: 10.1007/978-3-64220573-6_90.

[26] K. A. Abd Rashid et al., "A review on circular polarization antenna for wireless MIMO application," International Symposium on Technology Management and Emerging Technologies, Bandung, Indonesia, 2014, pp. 73-77, doi: 10.1109/ISTMET.2014.6936480.

[27] J. Wang, L. Zhaobiao, X. Li, "Analysis of MIMO Diversity Improvement Using Circular Polarized Antenna", International Journal of Antennas and Propagation, vol. 2014, Article ID 570923, doi.org/10.1155/2014/570923.

[28] U. Ullah, I. B. Mabrouk and S. Koziel, "Enhanced-Performance Circularly Polarized MIMO Antenna With Polarization/Pattern Diversity," IEEE Access, vol. 8, pp. 11887-11895, 2020, doi: 10.1109/ACCESS.2020.2966052.

[29] C. Balanis, Antenna Theory: Analysis: Analysis and Design, Willey, 2016.

[30] K. Mahmoud, M. El-Adawy, S. Ibrahem, R. Bansal and S. ZainudDeen, "A Comparison Between Circular and Hexagonal Array Geometries for Smart Antenna Systems Using Particle Swarm Optimization Algorithm," Progress In Electromagnetics Research, vol. 72, pp. 75-90, 2007, doi:10.2528/PIER07030904.

[31] C. Beckman and B. Lindmark, "The Evolution of Base Station Antennas for Mobile Communications," International Conference on Electromagnetics in Advanced Applications, Turin, Italy, 2007, pp. 8592, doi: 10.1109/ICEAA.2007.4387244.

[32] R. F. Harrington, Field Computation by Moment Methods, Wiley-IEEE Press, 1993.

[33] X. Chen, S. Zhang and Q. Li, "A Review of Mutual Coupling in MIMO Systems," IEEE Access, vol. 6, pp. 24706-24719, 2018, doi: 10.1109/ACCESS.2018.2830653. 